\title{
Genetically modified mesenchymal stem cells promote spinal fusion through polarized macrophages
}

\author{
Luchao $\mathrm{Yu}^{1,2,3}$, Qiang $\mathrm{Shi}^{1,3}$, Baokun Zhang ${ }^{1}$ and Jianguang $\mathrm{Xu}^{1 凶}$ \\ (c) The Author(s) 2021
}

\begin{abstract}
Spinal fusion is an effective treatment for low back pain and typically applied with prosthetic fixation devices. Spinal fusion can be improved by transplantation of mesenchymal stem cells (MSCs) into the paraspinal muscle. However, in contrast to the direct contribution of MSCs to spinal fusion, the indirect effects of MSCs on spinal infusion have not been studied and were thus addressed here. The correlation between the outcome of spinal fusion and the local macrophage number, polarization and the levels of placental growth factor (PIGF) in patients was analyzed. MSCs were genetically modified to overexpress PIGF, and its effects on macrophage proliferation and polarization were analyzed in vitro in a transwell co-culture system, as well as in vivo in a mouse model for spinal fusion, for which the cells were bilaterally injected into paravertebral muscles of the mouse lumbar spine. The effects on spinal fusion were assessed by microcomputed tomography and a custom four-point bending apparatus for structural bending stiffness. Local macrophages were analyzed by flow cytometry. We found that posterior spinal fusion could be improved by PIGF-expressing MSCs, compared to the control MSCs, evident by significant improvement of bone bridging of the targeted vertebrae. Mechanistically, PIGF-expressing MSCs appeared to attract macrophages and induce their M2 polarization, which in turn promotes the bone formation. Together, our data suggest that PIGF-expressing MSCs may improve spinal fusion through macrophage recruitment and polarization.
\end{abstract}

Laboratory Investigation (2022) 102:312-319; https://doi.org/10.1038/s41374-021-00693-4

\section{INTRODUCTION}

A great number of people who are younger than 45 years are suffered from severe low back pain, as a major reason for developing disability ${ }^{1}$. Most low back pain results from intervertebral disc degeneration as well as other pathological conditions such as scoliosis, spondylosis, spondylolisthesis, infection-associated inflammation, formation of cancer and posttraumatic fracture ${ }^{1}$. As an effective therapy of serious and sustained low back pain, spinal fusion of two or more adjacent vertebrae has been widely and routinely applied $^{2}$.

For a successful induction of spinal fusion, bone grafts and synthetic implants have been used. However, lack of efficiency and generation of side effects render researchers to identify alternative methods, e.g., of an injectable biological material agent to promote the formation of spinal infusion without the requirement for bone grafts and synthetic implants ${ }^{2}$. This approach can even avoid open surgery and lengthy hospitalization, which may greatly release the burden of patients both physiologically and financially ${ }^{3}$.

Some pilot studies have shown promising results. For example, direct delivery of osteogenic genes such as bone morphogenetic protein-2 (BMP-2), BMP-7 and BMP-9, has been shown to facilitate spinal fusion ${ }^{4-10}$. Moreover, induction of spinal infusion has been also achieved through injection of mesenchymal stem cells (MSCs) $^{11-22}$, leading to mechanical stabilization as the ultimate goal of spinal fusion ${ }^{23}$. MSCs are easily accessible in a non-invasive way. Moreover, MSCs possess a strong proliferative capacity to allow them to be nearly unlimitedly amplified in vitro ${ }^{11-22}$. However, the past studies have mainly focused on the direct contribution of MSCs to spinal fusion, while their indirect effects on spinal fusion have not been well investigated. It is noteworthy that there are a great number of evidence to show the role of MSCs in tissue repair, regeneration and remodeling relies on their crosstalk with local macrophages ${ }^{24-26}$. MSCs have been shown to regulate macrophage differentiation, proliferation and polarization to a phenotype called M2 (in contrast to traditional phagocytotic M1 macrophages) that are trophic and promote tissue regeneration through production and secretion of a number of growth factors ${ }^{27-29}$. However, the effects of MSCs on spinal fusion through macrophages have not been systemically studied.

Placental growth factor (PIGF) is a member of the vascular epithelial growth factor (VEGF) family, and plays a critical role in pathological angiogenesis ${ }^{30}$ and acts as a trigger for macrophage recruitment and differentiation ${ }^{29,31-35}$. The expression and function of PIGF in spinal fusion-associated macrophage infiltration have not been examined, and this question was thus addressed in the current study.

\section{MATERIALS AND METHODS}

Ethical approval, patient studies and experiment design

All experimental procedures have been approved by the research committee of Shanghai Jiaotong University Affiliated Sixth People's

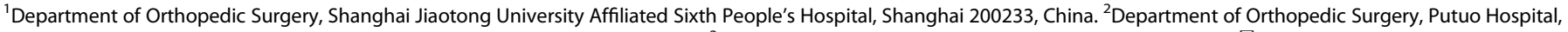
Shanghai University of Traditional Chinese Medicine, Shanghai 200062, China. ${ }^{3}$ These authors contributed equally: Luchao Yu, Qiang Shi. ${ }^{凶}$ email: mxj66447@yeah.net 
Hospital. The clinical studies included 22 patients with a diagnosis of non-pathological thoracolumbar burst fracture in the T12-L3 range (kyphos was above $20^{\circ}$ and/or anterior body collapse was $>50 \%$ ) and then received an internal fixation treatment at our hospital between 2015 and 2018. These patients included 14 male and 8 female, and the mean age at injury was 35.6 years $( \pm 11.8$, range $23-49)$. The Levels of the fracture were T12: 7, L1: 9, L2: 5 and L3: 1. The removal of the implants was between 6 and 8 months after the surgery. The biopsy was taken at the time of the implant removal. The RNA was extracted from the biopsy and analyzed for levels of some interested genes. The patients were invited to attend a clinical and radiographic review using a Low Back Outcome Score System (LBOSS) adapted from a previous publication ${ }^{36}$ and using an American Spinal Injury Association (ASIA) instruction to determine the nerve injury levels both pre-operatively and postoperatively. The AISA grade (A-E) has been determined by the following rule. AISA $A=$ no motor or sensory function is preserved in the sacral segments S4-S5. ASIA B = sensory but not motor function is preserved below the neurological level and includes the sacral segments S4-S5 ASIA $C=$ motor is preserved below the neurological level, and most of the key muscles below the neuro level have a muscle grade $<3$. ASIA D $=$ motor function is preserved below the neurological level, and at least half of key muscles below the neurological level have a muscle grade $=$ or $>3$. ASIA $E=$ NORMAL motor and sensory testing.

The mouse experiments were performed in accordance with guidance for the Care and Use of Laboratory Animals. Male and female 12-week-old C57BL/6 mice were purchased from SLAC Laboratory Animal Co. Ltd (Shanghai, China). Both male and female mice were used and distributed evenly in each experimental group. Mice were housed under a 12-hour light-dark cycle. For the in vivo mouse experiment, power calculations $(P<$ 0.05 ) were performed to include exactly sufficient animals for the observed effects to be legitimate. An allocation concealment method was used, and efforts were made to ensure that the potential confounders were minimized. No criteria were used for excluding animals (or experimental units) during the experiment, and no data were excluded during the analysis. The study did not have humane endpoints.

\section{Mouse MSCs and gene editting}

Mouse MSCs and naïve macrophages were isolated from bone marrow of isogeneic mice for in vivo expriments, as described $\mathrm{in}^{37} \mathrm{and}^{38}$, respectively. Mouse MSCs were cultured in StemXVivo Mesenchymal Stem Cell Expansion Medium (R\&D System, Los Angeles, CA, USA) and mouse macrophages were cultured in Iscove's Modified Dulbecco's Medium (IMDM, Thermo Scientific, Rockford, IL, USA) suppled with 15\% fetal bovine serum (FBS) in an incubator at a concentration of $5 \%$ carbon dioxide at $37^{\circ} \mathrm{C}$. MSC differentiation media were purchased from American Type Culture Collection (ATCC, Rockville, MD, USA). Transfection of MSCs by a full-length mouse PIGF2 construct a scrambled sequence (scr) as a control under a CMV promoter was performed with Lipofectamine 3000 reagent (Invitrogen, St. Louis, MO, USA). The phenotype of the modified PIGF-expressing MSCs were evaluated by flow cytometry analysis of the surface markers Sca-1, CD90, CD105, CD34, CD45 and HLA-DR, and by inducible differentiation of MSCs into osteocytes, adipocytes and chondrocytes, respectively, with different differentiation media (ATCC, Catalog number: PCS-500-052, PCS-500-050 and PCS-500-051). Alcian blue staining, Von kossa staining and Oil red O staining were applied to examine the differentiated chondrocytes, osteocytes and adipocytes, respectively.

\section{Animal treatment and grouping}

For in vivo injection of MSCs into the mouse spine, $2 \times 10^{6}$ (modified) MSCs were suspended in $30 \mu \mathrm{l}$ fibrin gel (FG, Sigma-Aldrich, St. Louis, MO, USA) and directly injected into the lumbar paravertebral muscle at L3-L5 after the mice were anesthetized by intraperitoneal injection of combined solution of $3 \mathrm{mg} / \mathrm{kg}$ xylazine and $100 \mathrm{mg} / \mathrm{kg}$ ketamine (Sigma-Aldrich). a blade cut of $1.5 \mathrm{~cm}$ long and $2 \mathrm{~mm}$ think was made on the middle back of the mice. For the first mouse experiment, the mice were assigned into 3 groups of 8 each: Group 1: mice received injection of MSCs-free FG; Group 2: mice received injection of FG containing MSCs-scr; Group 3: mice received injection of FG containing MSCs-PIGF. All the mice were kept for 6 weeks before analysis. For the second mouse experiment, the mice were assigned into 3 groups of 8 each: Group 1: mice received injection of FG containing MSCs-PIGF; Group 2: mice received injection of FG containing MSCs-PIGF and a weekly intravenous injection of $150 \mu \mathrm{l}$ liposome (Clodronateliposomes, Netherlands) afterwards; Group 3: mice received
Table 1. Low Back Outcome Score System for patients (LBOSSadapted*).

\begin{tabular}{|c|c|c|}
\hline Factor & Outcome & Points \\
\hline \multirow[t]{3}{*}{ Visual analogue scale of pain } & $7-10$ & 0 \\
\hline & $4-6$ & 4 \\
\hline & $0-3$ & 8 \\
\hline \multirow[t]{3}{*}{ Employment } & Unemployed & 0 \\
\hline & Part-time & 4 \\
\hline & Full-time & 8 \\
\hline \multirow{4}{*}{$\begin{array}{l}\text { Heavy social activities } \\
\text { (sports, etc) }\end{array}$} & None & 0 \\
\hline & Reduced & 3 \\
\hline & Modestly reduced & 6 \\
\hline & Back to previous level & 9 \\
\hline \multirow[t]{4}{*}{ Resting } & More than half day & 0 \\
\hline & Less than half day & 2 \\
\hline & Little rest & 4 \\
\hline & No need to rest & 6 \\
\hline \multirow[t]{4}{*}{ Live treatment } & More than once per day & 0 \\
\hline & Nearly everyday & 2 \\
\hline & Infrequently & 4 \\
\hline & Never & 6 \\
\hline \multirow[t]{3}{*}{ Sex life } & Severely affected & 0 \\
\hline & $\begin{array}{l}\text { Modestly or little } \\
\text { affected }\end{array}$ & 3 \\
\hline & unaffected & 6 \\
\hline \multirow{3}{*}{$\begin{array}{l}\text { Sleeping, walking and other } \\
\text { regular activities }\end{array}$} & Severely affected & 0 \\
\hline & $\begin{array}{l}\text { Modestly or little } \\
\text { affected }\end{array}$ & 3 \\
\hline & unaffected & 6 \\
\hline
\end{tabular}

*Range: 0-49; outcome: poor $\rightarrow$ good.

injection of FG containing MSCs-PIGF and a weekly intravenous injection of $150 \mu \mathrm{l}$ clodronate (Clodronateliposomes) afterwards. All the mice were kept for 6 weeks after the injection of FG into the lumbar paravertebral muscle.

\section{Co-culture of MSCs and macrophages}

For the co-cultre system, transwell chambers ( $8 \mu \mathrm{m}$ pore size, Corning Co. NY, USA) were sequentially added to $200 \mu \mathrm{l}$ of cells at a concentration of $10^{5} \mathrm{cells} / \mathrm{ml}$ and $500 \mu \mathrm{l}$ of FBS-free culture media for macrophages. After $24 \mathrm{~h}$, cells in the upper part of the small cell membrane were wiped off and determined. Arginase activity was measured with an arginase activity assay kit (MAK112, Sigma-Aldrich).

\section{Micro-CT analysis to assess bone formation}

Specimens were fixed in paraformaldehyde for $48 \mathrm{~h}$, after which microcomputed tomography

(MicroCT, mCT 40; Scanco Medical AG, Bassersdorf, Switzerland) examination was applied. Newly formed bone was separated from native bone by manual contouring. Some parameters were determined, including bone mineral density ( $\mathrm{mg}$ hydroxyapatite $(\mathrm{HA}) / \mathrm{cm}^{3}$ ) derived from the projectional image calculated with the calibration with $\mathrm{H}_{2} \mathrm{KPO}_{4}$ (Sigma-Aldrich) and the average bone thickness in micrometers. The lumbar spine rigidity was assessed by a four-point bending assay as described $^{23}$.

\section{Quantitative RT-PCR and ELISA}

The quantitative RT-PCR assay (RT-qPCR) was done routinely. Total RNA was extracted by a RNeasy kit (Qiagen, Valencia, CA, USA). Total RNA is transcribed by reverse transcription kit to generate cDNA for RT-qPCR 

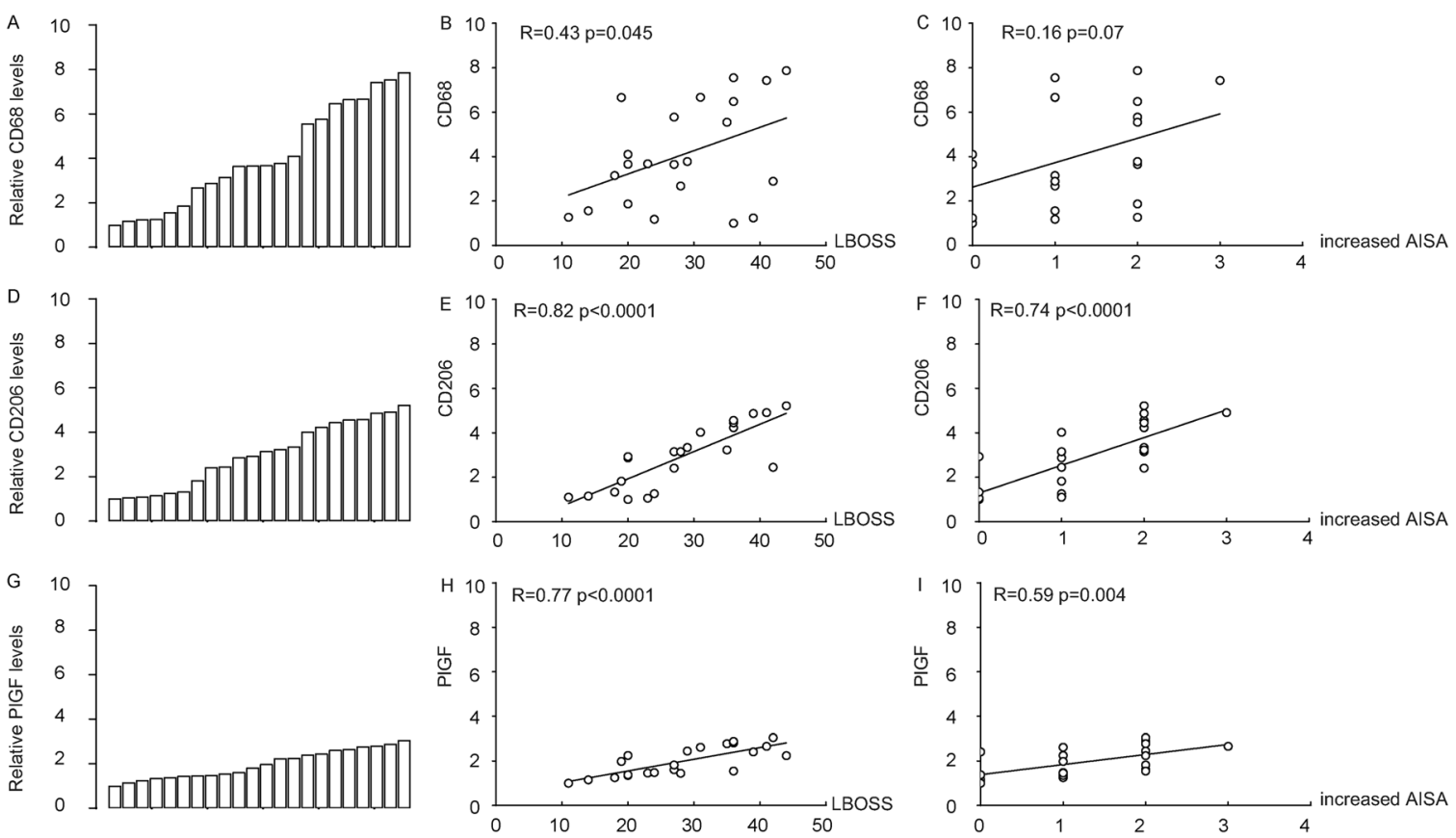

Fig. 1 Patient data suggest a possible role of macrophages in the recovery of the internal fusion after thoracolumbar fracture. A-F Some patients with a diagnosis of non-pathological thoracolumbar burst fracture in the T12-L3 range who have received an internal fixation treatment were selected in a clinical and radiographic review using a Low Back Outcome Score System (LBOSS) and the changes in the grade of AISA at post-operation compared to pre-operation. The biopsy at the injured regional muscle was used to assess the levels of CD68, CD206, and PIGF. A RT-qPCR for CD68. B Correlaton between LBOSS and CD68 levels. C Correlaton between AISA changes and CD68 levels. D RT-qPCR for CD206. E Correlaton between LBOSS and CD206 levels. F Correlaton between AISA changes and CD206 levels. G RT-qPCR for PIGF. H Correlaton between LBOSS and PIGF levels. I Correlaton between AISA changes and PIGF levels. $N=22$.

(Qiagen). All primers were purchased from Qiagen. GAPDH was used as an internal control for relative gene expression.

\section{Flow cytometry}

For flow cytometric analysis, macrophages and M2 macrophages were detected by $\mathrm{F} 4 / 80+$ and $\mathrm{F} 4 / 80+\mathrm{CD} 206+$, respectively. The analysis of the surface markers of the modified PIGF-expressing MSCs used fluorescinconjugated Sca-1, CD90, CD105, CD34, CD45 and HLA-DR antibodies (Becton-Dickinson Biosciences, San Jose, CA, USA). The proliferating cells were determined by analysis with a Ki-67 antibody (Thermo Scientific, San Jose, CA, USA) on fixed cells. Data were analyzed and presented by FlowJo software (Version 10, Flowjo LLC, Ashland, OR, USA).

\section{Statistical analysis}

All values represent the mean \pm standard deviation (SD). Statistical analysis of group differences was carried out using a one-way analysis of variance (ANOVA) test (GraphPad prism 7, GraphPad Software, Inc. La Jolla, CA, USA) followed by the Fisher's Exact Test to compare two groups. A value of $p<$ 0.05 was considered significant.

\section{RESULTS}

Patient data suggest a possible role of macrophages in the recovery of the internal fusion after thoracolumbar fracture Recent studies suggest a crosstalk between MSCs and macrophage in regulation of tissue repair and regeneration, and PIGF is likely a key factor to regulate macrophage recruitment, differentation and polarization. We thus studies the expression of macrophagespecific and macrophage-differentiation-specific markers to see whether it may be related to the outcome of the internal fusion of the patients. Some patients with a diagnosis of non-pathological thoracolumbar burst fracture in the T12-L3 range (kyphos was above $20^{\circ}$ and/or anterior body collapse was $>50 \%$ ) who have received an internal fixation treatment at our hospital between
2015 and 2018 were selected in a clinical and radiographic review using a Low Back Outcome Score System (LBOSS) adapted from a previous publication ${ }^{36}$, shown in Table 1, and using an ASIA grade to assess the nerve injury levels both pre-operatively and postoperatively. The biopsy at the injured regional muscle was used to assess the levels of macrophage infiltration by CD68, a panmacrophage marker, the levels of macrophage differentiation by CD206, a M2 or anti-inflammatory macrophage marker, and the levels of PIGF, a newly determined trigger for macrophage recruitment and differentiation. CD68 levels were determined by RT-qPCR (Fig. 1A), which showed strong correlation with the LBOSS of the patients $(p<0.05$; patients with better recovery appeared to have high macrophages at the injured/fusion site; Fig. 1B). The correlation between the CD68 levels with the AISA changes (every level improvement at post-operation compared to pre-operation is numbered 1, e.g., no improvement is $0 ; B$ to $C$ is $1 ; A$ to $C$ is 2 ) was also analyzed, showing a trend but not significant correlation $(p=$ 0.07 , Fig. 1C). CD206 levels were determined by RT-qPCR (Fig. 1D), which showed very strong correlation with the LBOSS of the patients $(p<0.001$; patients with better recovery appeared to have higher M2 macrophages at the injured/fusion site; Fig. 1E) and the AISA changes (Fig. 1F). PIGF levels were determined by RT-qPCR (Fig. 1G), which also showed very strong correlation with the LBOSS of the patients $(p<0.001$; patients with better recovery appeared to have higher PIGF levels at the injured/fusion site; Fig. $1 \mathrm{H}$ ) and the AISA changes (Fig. 1I). Together, these data suggest a possible positive effect of macrophages in the internal fusion of the vertebraes, which inspired us to assess it in the mouse model.

\section{Generation of MSCs expressing PIGF}

In order to assess the effects of MSCs on macrophage recruitment and differentation during spinal fusion, especially 

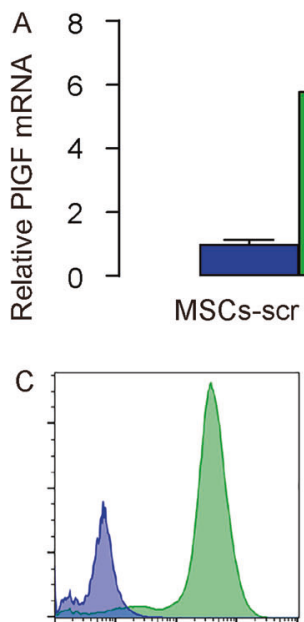

Sca-1

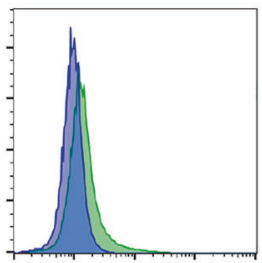

CD34
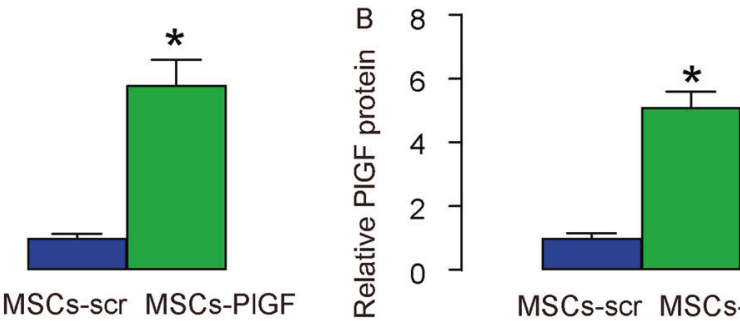

MSCs-scr MSCs-PIGF

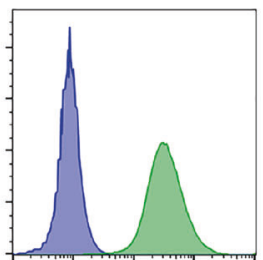

CD90

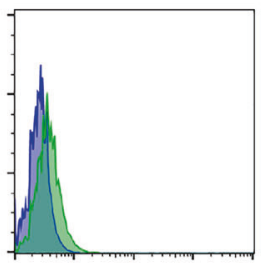

CD45

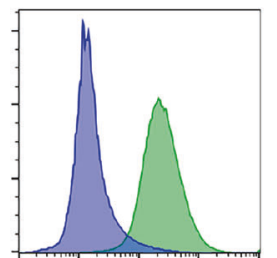

CD105

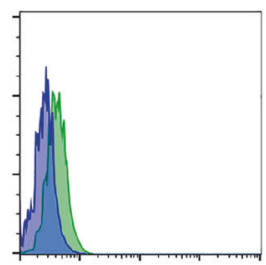

HLA-DR

$\mathrm{D}$
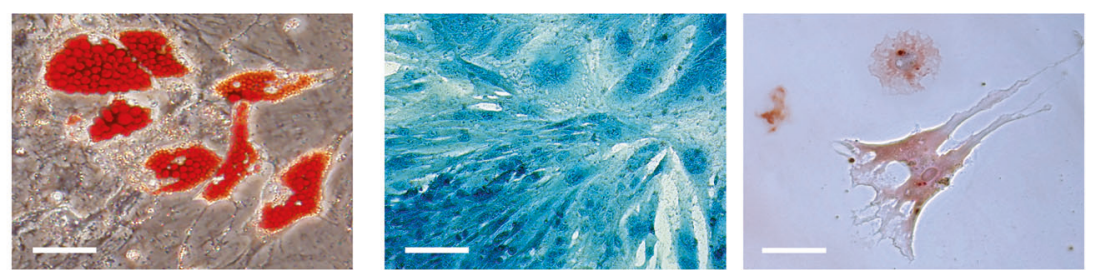

Fig. 2 Generation of MSCs expressing PIGF. We generated MSCs that overexpress PIGF (MSCs-PIGF), and MSCs that express a scramble sequence as a control (MSCs-scr). A-B PIGF levels by RT-qPCR (A), and by ELISA (B). C Flow cytometry for surface markers in MSCs-PIGF. D Oil red $O$ staining to evaluate adipogenic induction (left panel), alcian blue staining to evaluate chondrogenetic induction (middle panel), and Von kossa staining to evaluate osteogenic induction (right panel). ${ }^{*} p<0.05 . N=5$. Scale bars are $50 \mu \mathrm{m}$.

through PIGF, we generated MSCs that overexpress PIGF (MSCsPIGF), which could increase their potential to attract and polarize macrophages, as macrophages express the unique receptor, VEGF receptor 1, for PIGF. The increased PIGF in MSCs-PIGF, compared to control MSCs-scr, was confirmed by RT-qPCR (Fig. 2A), and by ELISA (Fig. 2B). The preservation of MSC characteristic by MSCs-PIGF was confirmed by expression of surface markers (Fig. 2C), and by maintanence of the differentating potentials (Fig. 2D).

\section{PIGF increases macrophage migratory potential and M2- differentiation by MSCs}

Next, we assessed the effects of expression of PIGF in MSCs on macrophages in a co-culture system. We found that PIGF expression in MSCs significantly increased the invasion and migration of co-cultured macrophages (Fig. 3A-C). Moreover, PIGF expression in MSCs significantly decreased the expression of M1/ proinflammatory genes (iNOS, TNFa, IL-6 and IL-1ß) and significantly increased the expression of $M 2$ /anti-inflammatory genes (Arginase 1 (ARG1), CD163, CD206, Fizz1 and Ym1) in the cocultured macrophages (Fig. 3D). The PIGF-induced M2-like differentation of the co-cultured macrophages was furhter confirmed by analysis on M2 surface marker CD206 (Fig. 3E-F) and by increases in arginase activity (Fig. 3G). However, the total macrophage number in the co-cultured system was not significantly altered (Fig. 3G-I). Together, these data suggest that PIGF in MSCs increases macrophage migratory potential and M2differentiation without altering macrophage growth.

\section{PIGF expression in MSCs promote spinal fusion in vivo}

The effects of PIGF expression in MSCs on spinal fusion were tested in a mouse model. The mice were assigned into 3 groups of 8 each: Group 1: mice received injection of MSCs-free FG; Group 2: mice received injection of FG containing MSCs-scr; Group 3: mice received injection of FG containing MSCs-PIGF. All the mice were kept for 6 weeks before analysis. We found that FG alone did not induce spinal fusion, while MSCs in FG induced a few spinal fusion, but MSCs-PIGF in FG induced significanly more spinal fusion (Fig. 4A). Bone mineral density was quantified, showing the highest in FG, while reduced in FG + MSCs-scr and further reduced in FG + MSCs-PIGF (Fig. 4B), which was consistent with the new bone formation (Fig. 4A). While average bone thickness among 3 groups were not significantly different (Fig. 4C), the spinal regidity between $\mathrm{FG}+\mathrm{MSCs}-\mathrm{scr}$ and $\mathrm{FG}+\mathrm{MSC}-\mathrm{PIGF}$ groups was not significantly different (Fig. 4D), suggesting that the promotion of vertebrae fusion is not compensated by altering spinal regidity. Thus, PIGF expression in MSCs promote spinal fusion in vivo. 

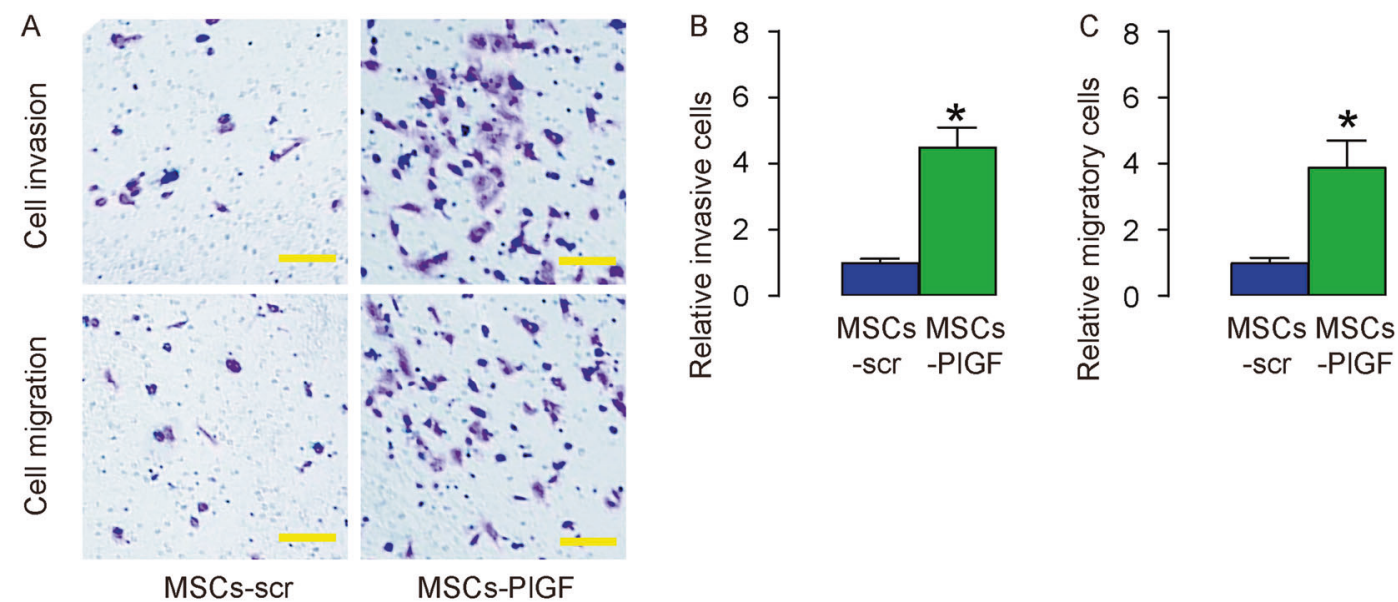

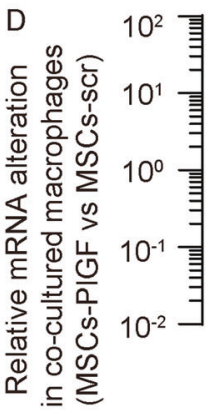
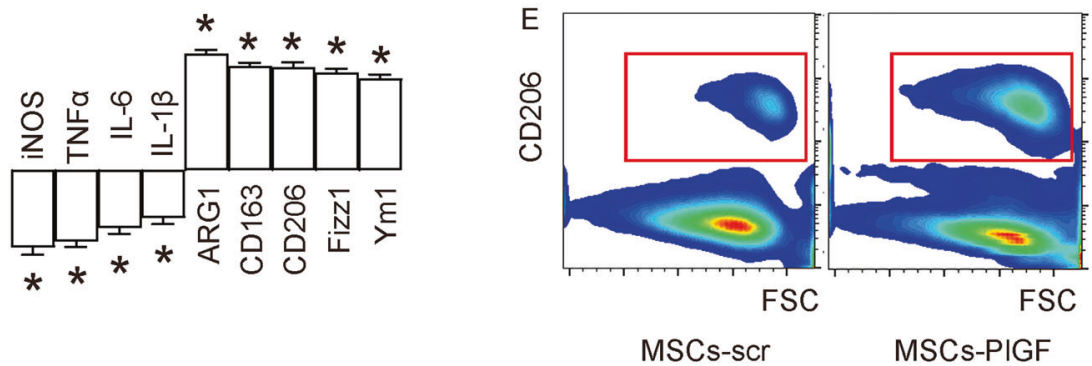
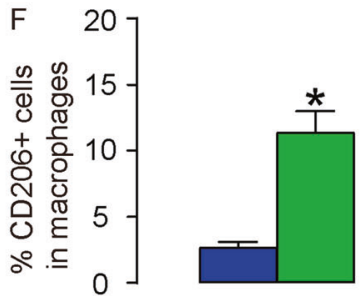

MSCs MSCs -scr -PIGF

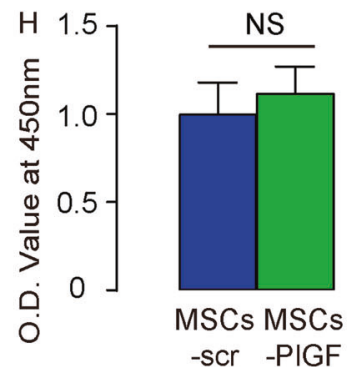

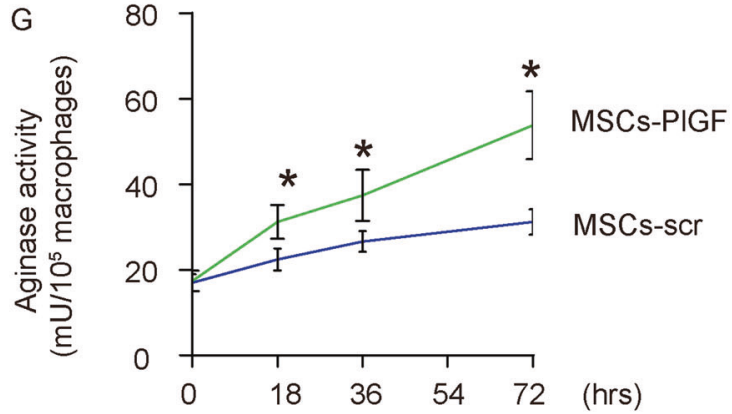

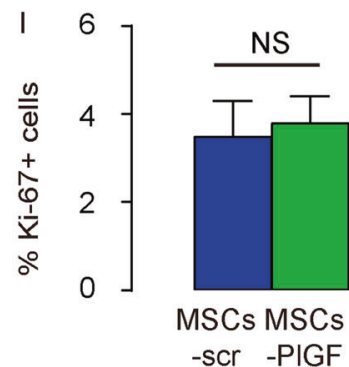

Fig. 3 PIGF increases macrophage migratory potential and M2-differentiation by MSCs. A-C Transwell cell invasion assay and cell migraton assay, shown by representatitve images (A), and by quantification of invasive cells (B) and quantification of migratory cells (C). D RT-qPCR for genes in macrophages co-cultured with MSCs-PIGF versus macrophages co-cultured with MSCs-scr. E-F Flow cytometry for CD206 in macrophages co-cultured with MSCs-PIGF versus macrophages co-cultured with MSCs-scr, shown by representative flow charts (E) and by quantification (F). G Arginase activity assay. H Quantification of cell number of a CCK-8 assay. I Quantification of Ki-67+ cells by flow cytometry. ${ }^{*} p<0.05$. NS non-significant. $N=5$. Scale bars are $50 \mu \mathrm{m}$.

\section{PIGF expression in MSCs promote spinal fusion through macrophages}

We examined the mechanisms underlying the enhancement of spinal fusion by PIGF-expressing MSCs. Analysis of the macrophage number in the fusion site showed signficantly increases in
F4/80+ macrophages in FG + MSCs-scr mice, compared to FG mice, and this increase in macrophages further increased in the FG + MSCs-PIGF mice, shown by representative flow charts (Fig. 5A) and by quantification (Fig. 5B). In order to assess whether the recruitment of macrophages is the reason for the 

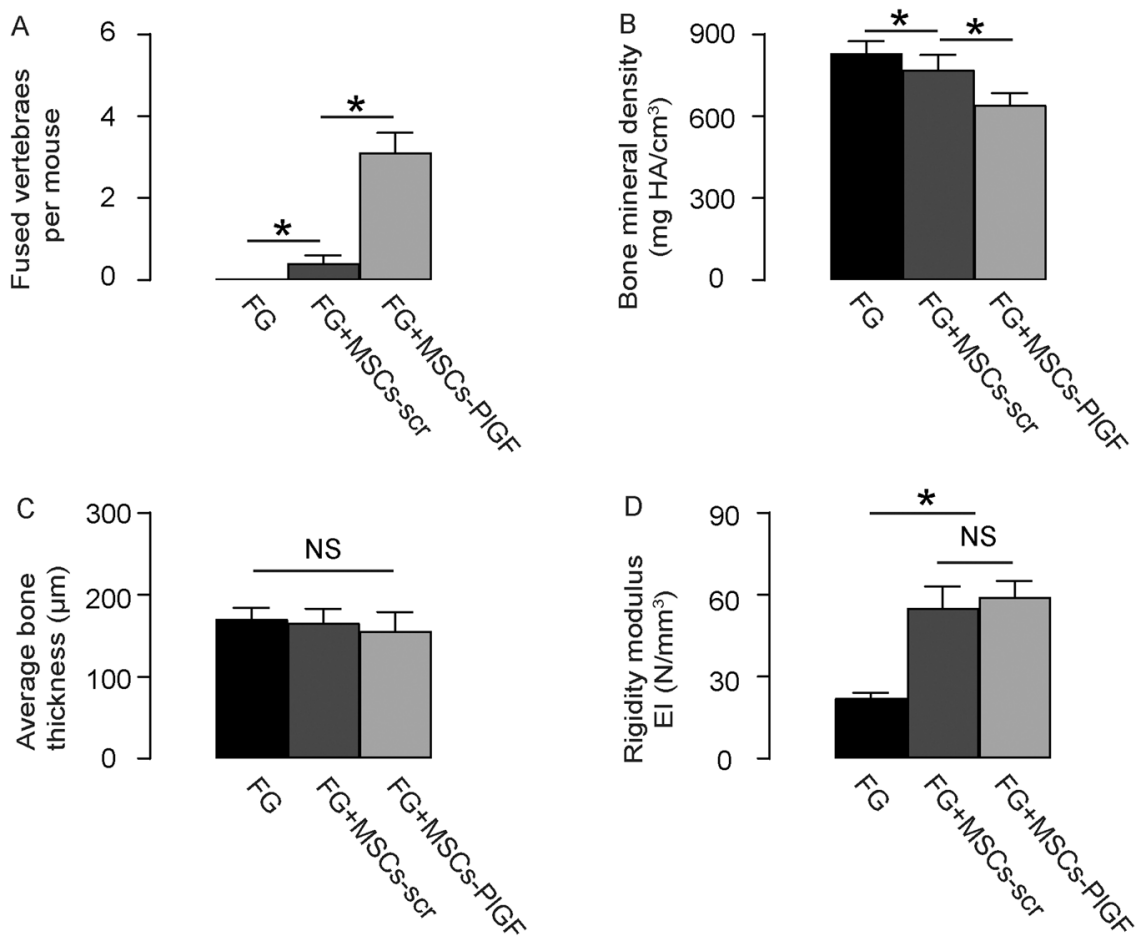

Fig. 4 PIGF expression in MSCs promote spinal fusion in vivo. The effects of PIGF expression in MSCs on spinal fusion were tested in a mouse model. The mice were assigned into 3 groups of 8 each: Group 1: mice received injection of MSCs-free FG; Group 2: mice received injection of FG containing MSCs-scr; Group 3: mice received injection of FG containing MSCs-PIGF. All the mice were kept for 6 weeks before analysis. A Quantifiation of spinal fusion by fused vertebrae number. B Bone mineral density. C Average bone thickness. D Bone rigidity by El. ${ }^{*} p<0.05$. NS non-significant. $N=8$.

enhancement of spinal fusion by by PIGF-expressing MSCs, we performed another experiment, in which macrophages were chemically depleted by clodronate ${ }^{39}$. For this experiment, the mice were assigned into 3 groups of 8 each: Group 1: mice received injection of FG containing MSCs-PIGF; Group 2: mice received injection of FG containing MSCs-PIGF and a weekly intravenous injection of liposome, the control for clodronate, till the end of the 6-weeks followup; Group 3: mice received injection of FG containing MSCs-PIGF and a weekly intravenous injection of clodronate to deplete macrophages. We found that macrophagedepletion by clodronate abolished the effects of PIGF in MSCs on spinal fusion (Fig. 5C), and the macrophage depletion in clodronate-treated mice was confirmed by flow cytometry (Fig. 5D-E). Thus, PIGF expression in MSCs promote spinal fusion through macrophages.

\section{DISCUSSION}

Previous studies have demonstrated a seminal role of macrophages in the repair of the injured spinal $\operatorname{cord}^{40}$. These macrophages are mainly derived from circulating monocytes from bone marrow and spleen ${ }^{40}$. The injured tissue secretes cytokines and chemokines into circulation as chemoattractants for recruiting these monocytes into the site where they differentiate into macrophages to perform multiple functions involved in the wound healing process ${ }^{41}$. Although these studies have well defined a pivotal role of macrophages in the spinal repair, a similar role of macrophages in the MSCs-mediated spinal fusion has not been analyzed.

The total macrophages are contributed by $\mathrm{M} 1$ and $\mathrm{M} 2$ macrophages. Since we detected a strong correlation between LBOSS/ AISA with the M2 marker CD206, but only a modest or no correlation between the total macrophage marker CD68 (M1 + M2) and LBOSS or AISA, respectively, which may be due to the much more important effect of $M 2$ macrophages than M1 macrophages. M2 macrophages are the macrophage subtypes associated with tissue repair and regeneration through their production and secretion of a number of trophic cytokines. Moreover, the strong correlation between PIGF and the recovery suggests PIGF as a key factor to recruit macrophages, as reported by many studies in other area $29,31-34,42-46$. On the other hand, presence of M1 macrophages could have neutral or even negative effects on LBOSS, while the difference in $\mathrm{M} 1$ macrophage number/ratio to total macrophages in addition to the number of M2 macrophages may cause the variations of LBOSS.

In our in vitro co-culture experiment, PIGF expression in MSCs significantly increased the invasion and migration of co-cultured macrophages and polarized macrophages to a M2-like phenotype, suggesting that PIGF has a dual role in the regulation of spinal fusion by macrophages. First, macrophages are recruited to the site through being attracted to PIGF through its expression of VEGFR1. Second, the signaling cascades downstream of PIGF/NEGFR1 regulatory axis may activate the differentiation process in macrophages, perhaps through STAT1/STAT3/STAT6 signaling as reported $^{47-49}$, to promote the bone formation during spinal fusion.

In our in vivo experiment, the depletion of macrophages by clodronate was sufficient to antagonize the effects of overexpressing PIGF in MSCs, again supporting the hypothesis that PIGF expression in MSCs promotes spinal fusion by macrophages. Interestingly, the use of clodronate not only depleted most of macrophages, but also seemed to preferably deplete all M2 macrophages. This result may be resulting from the different properties of $M 1$ and $M 2$ macrophages in the phagocytosis of clodronate or from the difference in their survival potential after engulfing clodronate ${ }^{39,50,51}$. The effects of PIGF-expressing MSCs were completely abolished by use of clodronate, suggesting that 


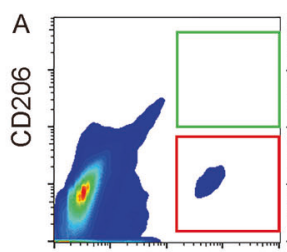

F $4 / 80$

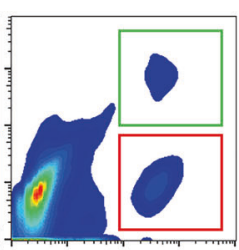

F $4 / 80$

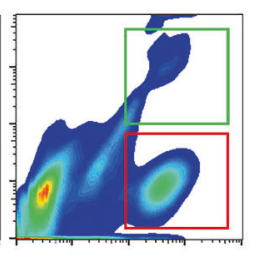

$\mathrm{F} 4 / 80$

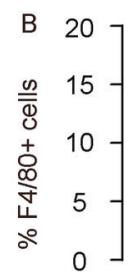

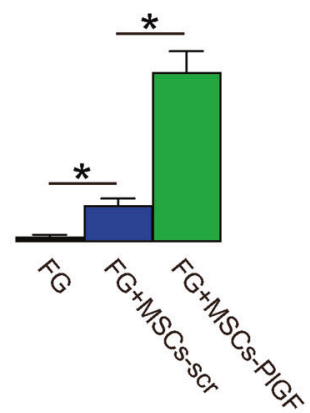

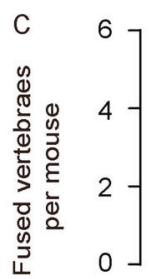

FG+MSCs-scr

FG+MSCs-PIGF

FG
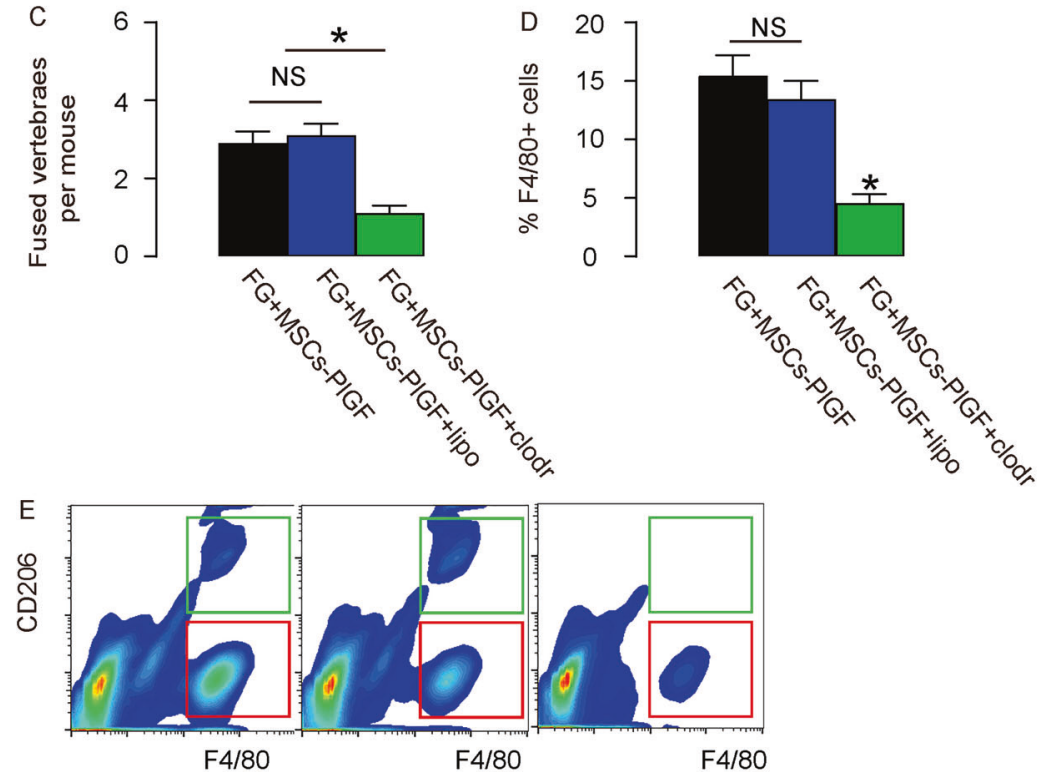

FG+MSCs-PIGF FG+MSCs-PIGF+lipo FG+MSCs-PIGF+clodr

Fig. 5 PIGF expression in MSCs promote spinal fusion through macrophages. A, B Flow cytometry analysis of the macrophage number in the fusion site, shown by representative flow charts (A) and by quantification (B). C-E In order to assess whether the recruitment of macrophages is the reason for the enhancement of spinal fusion by by PIGF-expressing MSCs, macrophages were chemically depleted by clodronate. For this experiment, the mice were assigned into 3 groups of 8 each: Group 1: mice received injection of FG containing MSCs-PIGF; Group 2: mice received injection of FG containing MSCs-PIGF and a weekly intravenous injection of liposome, the control for clodronate, till the end of the 6-weeks followup; Group 3: mice received injection of FG containing MSCs-PIGF and a weekly intravenous injection of clodronate to deplete macrophages. C Quantifiation of spinal fusion by fused vertebrae number. D-E Flow cytometry analysis of the macrophage number in the fusion site, shown by quantification (D), and by representative flow charts (E). ${ }^{*} p<0.05$. NS non-significant. $N=8$.

the major effective target of PIGF should be macrophages, rather than endothelial cells, since they are the two major cell types that express the unique receptor for PIGF on site, and clodronate does not affect endothelial cells at all ${ }^{30}$.

To summarize, here using both in vitro and in vivo tools, we were able to demonstrate a critical role of macrophages in the spinal fusion enhanced by transplantation of MSCs. Moreover, we found that PIGF could be a promising target gene to manipulate for regulating macrophage recruitment and differentiation at the fusion site.

\section{REFERENCES}

1. Manfre, L., Van Goethem, J. Low Back Pain. In: Hodler, J., Kubik-Huch, R. A., von Schulthess, G. K. (eds.) Diseases of the Brain, Head and Neck, Spine 2020-2023: Diagnostic Imaging: Cham (CH), pp 225-230 (2020).

2. Willems, P. C. Provocative diskography: safety and predictive value in the outcome of spinal fusion or pain intervention for chronic low-back pain. J. Pain Res. 7, 699-705 (2014).

3. Willems, P. C., Staal, J. B., Walenkamp, G. H. \& de Bie, R. A. Spinal fusion for chronic low back pain: systematic review on the accuracy of tests for patient selection. Spine J. 13, 99-109 (2013).

4. Chidambaran, V., Pilipenko, V., Jegga, A. G., Geisler, K. \& Martin, L. J. Systems Biology guided gene enrichment approaches improve prediction of chronic postsurgical pain after spine fusion. Front. Genet. 12, 594250 (2021).
5. Zhu, W. et al. Combined bone morphogenetic protein-2 and -7 gene transfer enhances osteoblastic differentiation and spine fusion in a rodent model. J. Bone Miner Res. 19, 2021-2032 (2004).

6. Yoon, S. T. \& Boden, S. D. Spine fusion by gene therapy. Gene Ther 11, 360-367 (2004).

7. Hidaka, C., Goshi, K., Rawlins, B., Boachie-Adjei, O. \& Crystal, R. G. Enhancement of spine fusion using combined gene therapy and tissue engineering BMP-7expressing bone marrow cells and allograft bone. Spine (Phila Pa 1976) 28, 2049-2057 (2003).

8. Cha, C. W. \& Boden, S. D. Gene therapy applications for spine fusion. Spine (Phila Pa 1976) 28(15 Suppl), S74-S84 (2003).

9. Boden, S. D. et al. Lumbar spine fusion by local gene therapy with a CDNA encoding a novel osteoinductive protein (LMP-1). Spine (Phila Pa 1976) 23, 2486-2492 (1998).

10. Morone M. A., et al. The Marshall R. Urist Young Investigator Award. Gene expression during autograft lumbar spine fusion and the effect of bone morphogenetic protein 2. Clin. Orthop. Relat. Res. 351, 252-265 (1998).

11. Robbins, M. A., Haudenschild, D. R., Wegner, A. M. \& Klineberg, E. O. Stem cells in spinal fusion.Global Spine J. 7, 801-810 (2017).

12. Lee, $S$. et al. Brief report: human perivascular stem cells and Nel-Like Protein1 synergistically enhance spinal fusion in osteoporotic rats. Stem Cells 33, 3158-3163 (2015).

13. Chung, C. G. et al. Human perivascular stem cell-based bone graft substitute induces rat spinal fusion. Stem Cells Transl. Med. 4, 538 (2015).

14. Chung, C. G. et al. Human perivascular stem cell-based bone graft substitute induces rat spinal fusion. Stem Cells Transl. Med. 3, 1231-1241 (2014). 
15. Lee, T. H. et al. Characterization and spinal fusion effect of rabbit mesenchymal stem cells. BMC Res. Notes 6, 528 (2013).

16. McIntosh, K. R. et al. Immunogenicity of allogeneic adipose-derived stem cells in a rat spinal fusion model. Tissue Eng Part A 15, 2677-2686 (2009).

17. Gottfried, O. N. \& Dailey, A. T. Mesenchymal stem cell and gene therapies for spinal fusion. Neurosurgery 63, 380-391 (2008). discussion 391-382.

18. Miyazaki, M. et al. Comparison of human mesenchymal stem cells derived from adipose tissue and bone marrow for ex vivo gene therapy in rat spinal fusion model. Spine (Phila Pa 1976) 33, 863-869 (2008).

19. Sheyn, D. et al. Nonvirally engineered porcine adipose tissue-derived stem cells: use in posterior spinal fusion. Stem Cells 26, 1056-1064 (2008).

20. Hasharoni, A. et al. Murine spinal fusion induced by engineered mesenchymal stem cells that conditionally express bone morphogenetic protein-2. J Neurosurg Spine 3, 47-52 (2005).

21. Cinotti, G. et al. Experimental posterolateral spinal fusion with porous ceramics and mesenchymal stem cells. J Bone Joint Surg Br 86, 135-142 (2004).

22. Dumont, R. J. et al. Ex vivo bone morphogenetic protein-9 gene therapy using human mesenchymal stem cells induces spinal fusion in rodents. Neurosurgery 51, 1239-1244 (2002). discussion 1244-1235.

23. Sheyn, D. et al. Genetically modified mesenchymal stem cells induce mechanically stable posterior spine fusion. Tissue Eng Part A 16, 3679-3686 (2010).

24. Zhang, J. et al. Interleukin-4-loaded hydrogel scaffold regulates macrophages polarization to promote bone mesenchymal stem cells osteogenic differentiation via TGF-beta1/Smad pathway for repair of bone defect. Cell Prolif. 53, e12907 (2020).

25. Yu, B., Alboslemy, T., Safadi, F. \& Kim, M. H. Glycoprotein Nonmelanoma Clone B Regulates the Crosstalk between Macrophages and Mesenchymal Stem Cells toward Wound Repair. J. Invest. Dermatol. 138, 219-227 (2018).

26. Wang, M. et al. Crosstalk of mesenchymal stem cells and macrophages promotes cardiac muscle repair. Int. J. Biochem. Cell Biol. 58, 53-61 (2015).

27. Shin, R. L., Lee, C. W., Shen, O. Y., Xu, H. \& Lee, O. K. The crosstalk between mesenchymal stem cells and macrophages in bone regeneration: a systematic review. Stem Cells Int. 2021, 8835156 (2021).

28. Xu, C., Fu, F., Li, X. \& Zhang, S. Mesenchymal stem cells maintain the microenvironment of central nervous system by regulating the polarization of macrophages/ microglia after traumatic brain injury. Int. J. Neurosci. 127, 1124-1135 (2017).

29. Yang, W., et al. Placental growth factor in beta cells plays an essential role in gestational beta-cell growth. BMJ Open Diabetes Res. Care 8, e000921 (2020).

30. Dewerchin, M., Carmeliet, P. PIGF: a multitasking cytokine with disease-restricted activity. Cold Spring Harb Perspect Med. 2, a011056 (2012).

31. He, C. et al. Placental growth factor mediates crosstalk between lung cancer cells and tumor-associated macrophages in controlling cancer vascularization and growth. Cell Physiol. Biochem. 47, 2534-2543 (2018).

32. Adini, A., Kornaga, T., Firoozbakht, F. \& Benjamin, L. E. Placental growth factor is a survival factor for tumor endothelial cells and macrophages. Cancer Res. 62 , 2749-2752 (2002).

33. Li, X. et al. Placental growth factor contributes to liver inflammation, angiogenesis, fibrosis in mice by promoting hepatic macrophage recruitment and activation. Front. Immunol. 8, 801 (2017).

34. Khurana, R. et al. Placental growth factor promotes atherosclerotic intimal thickening and macrophage accumulation. Circulation 111, 2828-2836 (2005).

35. Bacsi, A. et al. Placental macrophage contact potentiates the complete replicative cycle of human cytomegalovirus in syncytiotrophoblast cells: role of interleukin-8 and transforming growth factor-beta1. J. Interferon. Cytokine Res. 19, 1153-1160 (1999).

36. Greenough, C. G. \& Fraser, R. D. Assessment of outcome in patients with low-back pain. Spine (Phila Pa 1976) 17, 36-41 (1992).

37. Boregowda, S. V., Krishnappa, V. \& Phinney, D. G. Isolation of mouse bone marrow mesenchymal stem cells. Methods Mol. Biol. 1416, 205-223 (2016).

38. Modderman, W. E., Vrijheid-Lammers, T., Lowik, C. W. \& Nijweide, P. J. Removal of hematopoietic cells and macrophages from mouse bone marrow cultures: isolation of fibroblastlike stromal cells. Exp. Hematol. 22, 194-201 (1994).

39. Camilleri, J. P. et al. Methods for assessing splenic macrophage depletion by liposome encapsulated clodronate. Inflamm. Res. 44, 152-157 (1995).

40. Milich, L. M., Ryan, C. B. \& Lee, J. K. The origin, fate, and contribution of macrophages to spinal cord injury pathology. Acta Neuropathol. 137, 785-797 (2019).

41. Das, A. et al. Monocyte and macrophage plasticity in tissue repair and regeneration. Am. J. Pathol. 185, 2596-2606 (2015).

42. Zhou, X. \& Qi, Y. Larynx carcinoma regulates tumor-associated macrophages through PLGF signaling. Sci. Rep. 5, 10071 (2015).
43. Raevens, S. et al. Placental growth factor inhibition targets pulmonary angiogenesis and represents a therapy for hepatopulmonary syndrome in mice. Hepatology 68, 634-651 (2018).

44. Heindryckx, F. et al. The placental growth factor as a target against hepatocellular carcinoma in a diethylnitrosamine-induced mouse model. J. Hepatol. 58, 319-328 (2013).

45. Pavlov, O. V., Sheveleva, T. S. \& Selkov, S. A. In vitro expression of vascular endothelial growth factor and its receptors by placental macrophages. Bull Exp. Biol. Med. 153, 222-225 (2012).

46. Maes, C. et al. Placental growth factor mediates mesenchymal cell development, cartilage turnover, and bone remodeling during fracture repair. J. Clin. Invest. 116, 1230-1242 (2006).

47. Hou, Y., Shi, G., Guo, Y. \& Shi, J. Epigenetic modulation of macrophage polarization prevents lumbar disc degeneration. Aging (Albany N Y) 12, 6558-6569 (2020).

48. Ivashkiv, L. B. Epigenetic regulation of macrophage polarization and function. Trends Immunol. 34, 216-223 (2013).

49. Takeuch, O. \& Akira, S. Epigenetic control of macrophage polarization. Eur. J. Immunol. 41, 2490-2493 (2011).

50. van Rooijen, N. \& van Nieuwmegen, R. Elimination of phagocytic cells in the spleen after intravenous injection of liposome-encapsulated dichloromethylene diphosphonate. An enzyme-histochemical study. Cell Tissue Res. 238, 355-358 (1984).

51. Claassen, E. \& Van Rooijen, N. The effect of elimination of macrophages on the tissue distribution of liposomes containing [3H]methotrexate. Biochim. Biophys. Acta 802, 428-434 (1984).

\section{ACKNOWLEDGEMENTS}

This study was supported by Natural Science Foundation of China (NO: 81672217).

\section{AUTHOR CONTRIBUTIONS}

L.Y., Q.S., B.Z. and J.X. are responsible for data acquisition and analysis; L.Y., Q.S. and J.X. are responsible for study conception and design; L.Y. drafted the manuscript. All authors agree to the publication of the study. J.X. is responsible for funding and the guarantee of the study.

\section{COMPETING INTERESTS}

The authors declare no competing interests.

\section{ADDITIONAL INFORMATION}

Correspondence and requests for materials should be addressed to Jianguang Xu.

Reprints and permission information is available at http://www.nature.com/ reprints

Publisher's note Springer Nature remains neutral with regard to jurisdictional claims in published maps and institutional affiliations. ation, distribution and reproduction in any medium or format, as long as you give appropriate credit to the original author(s) and the source, provide a link to the Creative Commons license, and indicate if changes were made. The images or other third party material in this article are included in the article's Creative Commons license, unless indicated otherwise in a credit line to the material. If material is not included in the article's Creative Commons license and your intended use is not permitted by statutory regulation or exceeds the permitted use, you will need to obtain permission directly from the copyright holder. To view a copy of this license, visit http://creativecommons. org/licenses/by/4.0/.

(c) The Author(s) 2021 